\title{
Intensity Noise Transfer Through a Diode-pumped Titanium Sapphire Laser System
}

Tawfieq, Mahmoud; Hansen, Anders Kragh; Jensen, Ole Bjarlin; Marti, Dominik; Sumpf, Bernd; Andersen, Peter E.

Published in:

IEEE Journal of Quantum Electronics

Link to article, DOI:

10.1109/JQE.2017.2777860

Publication date:

2018

Document Version

Peer reviewed version

Link back to DTU Orbit

Citation (APA):

Tawfieq, M., Hansen, A. K., Jensen, O. B., Marti, D., Sumpf, B., \& Andersen, P. E. (2018). Intensity Noise Transfer Through a Diode-pumped Titanium Sapphire Laser System. IEEE Journal of Quantum Electronics, 54(1), [1700209 ]. https://doi.org/10.1109/JQE.2017.2777860

\section{General rights}

Copyright and moral rights for the publications made accessible in the public portal are retained by the authors and/or other copyright owners and it is a condition of accessing publications that users recognise and abide by the legal requirements associated with these rights.

- Users may download and print one copy of any publication from the public portal for the purpose of private study or research.

- You may not further distribute the material or use it for any profit-making activity or commercial gain

- You may freely distribute the URL identifying the publication in the public portal 


\title{
Intensity Noise Transfer Through a Diode- pumped Titanium Sapphire Laser System
}

\author{
Mahmoud Tawfieq, Anders Kragh Hansen, Ole Bjarlin Jensen, Dominik Marti, \\ Bernd Sumpf, and Peter E. Andersen
}

\begin{abstract}
In this paper, we investigate the noise performance and transfer in a titanium sapphire (Ti:S) laser system. This system consists of a DBR tapered diode laser, which is frequency doubled in two cascaded nonlinear crystals and used to pump the Ti:S laser oscillator. This investigation includes electrical noise characterizations of the utilized power supplies, the optical noise of the fundamental light, the second harmonic light, and finally the optical noise of the femtosecond pulses emitted by the Ti:S laser. Noise features originating from the electric power supply are evident throughout the whole transfer chain. It is demonstrated that improving the electrical noise provides an easy method for reducing the relative intensity noise (RIN) in all stages. The frequency doubled light is shown to have a higher RIN than the fundamental light. In particular, the cascaded system is seen to exhibit higher RIN than a setup with only a single nonlinear crystal. The Ti:S is shown to have a cut-off frequency around $500 \mathrm{kHz}$, which means that noise structures of the pump laser above this frequency are strongly suppressed. Finally, the majority of the Ti:S noise seems to originate from the laser itself, which partly can be explained by the effect of relaxation oscillation frequency.
\end{abstract}

Index Terms - Laser noise, Noise measurement, Nonlinear optics, Laser cavity resonators.

\section{INTRODUCTION}

L ASER NOISE has been of high interest for many years, both in the context of fundamental physics and for a variety of laser applications. Single-frequency lasers constitute cases of strong interest in quantum optics which have been studied in great detail [1]-[3]. The obtained understanding has also brought significant benefits for the further development of lasers with favourable noise properties, as required for many different applications [4]-[6]. While these applications are mainly within fiber-optic communication, noise is an equivalently important factor within other fields such as bioimaging [7]-[9]. A good understanding of how laser noise is

This research was supported by the European project FAMOS (FP7 ICT 317744).

Mahmoud Tawfieq was at the time of this study with the Department of Photonics Engineering, Technical University of Denmark, 4000 Roskilde, Denmark. He is now, together with Bernd Sumpf at the Ferdinand-BraunInstitut, Leibniz-Institut für Höchstfrequenztechnik, 12489 Berlin, Germany (e-mail: Mahmoud.Tawfieq@fbh-berlin.de, Bernd.Sumpf@fbh-berlin.de).

Anders Kragh Hansen, Ole Bjarlin Jensen, Dominik Marti, and Peter E. Andersen are with the Department of Photonics Engineering, Technical University of Denmark, 4000 Roskilde, Denmark (e-mail: ankrh@fotonik.dtu.dk, ojen@fotonik.dtu.dk, domar@fotonik.dtu.dk, peta@fotonik.dtu.dk). generated and transferred does therefore not only help improve the performance of particular devices, but also to realize systems with performances close to the fundamental noise limitations.

One of these devices is the tapered diode laser [10], where the noise performance is decisive in regard to its performance in several applications. These light sources have over the last decade found a strong foothold in various applications, especially within the field of nonlinear frequency conversion. One of these nonlinear processes is the second harmonic generation (SHG) [11],[12]. This process offers an efficient method for generation of coherent light in the blue-green spectral range, where efficient, direct, and diffraction limited emission from semiconductor materials does not exist in the watt-range [13]. Such frequency converted laser systems have been used as pump sources for titanium sapphire (Ti:S) lasers and examples of their use within biophotonics imaging, in the field of retinal optical coherence tomography (OCT) and in multi photon tomography (MPT) has been reported [14]-[16].

In this study, we identify the origin of noise and the mechanisms of noise transfer in an optical system suitable for among others, biomedical imaging. This system consists of a DBR tapered diode laser, the emission of which is frequency doubled through two subsequent nonlinear crystals and then used to pump a Ti:S laser. The motivation is thus to understand the noise performance and transfer of such a system including the electrical noise of the power supplies used, the optical noise of the tapered diode laser, its frequency doubling, and finally the noise performance of the short pulses emitted by the Ti:S laser. Thus the motivation of this work is not to obtain a reduction of the laser noise, but will rather serve as a step toward understanding the relationship between the laser noise and its transfer into noise sensitive Ti:S applications.

\section{Light SOURCE}

The setup of the investigated optical system is conceptually sketched in Fig. 1 and is very similar to the setup described in [12]. The laser is a $6 \mathrm{~mm}$ long distributed Bragg-reflector (DBR) tapered diode laser, consisting of an unpumped $1 \mathrm{~mm}$ DBR section, a $1 \mathrm{~mm}$ ridge waveguide (RW) followed by a $4 \mathrm{~mm}$ long tapered power amplifier (TPA) section, where the RW and TPA sections have separate electrical contacts. The laser emits at a wavelength of $1063 \mathrm{~nm}$, with an output power of more than $10.5 \mathrm{~W}$, when operated at $20^{\circ} \mathrm{C}$, at a ridge 
section current of $300 \mathrm{~mA}$, and a taper section current of $14 \mathrm{~A}$. The laser beam is collimated and passed through an optical isolator ( $60 \mathrm{~dB}$ isolation), after which the beam is focused into a nonlinear crystal for second harmonic generation. In order to increase the conversion efficiency, the frequency doubled and residual infrared light are refocused into a second nonlinear crystal. $2.8 \mathrm{~W}$ of diffraction limited green light is generated in this so-called cascade of nonlinear crystals. After the frequency doubling, the residual infrared light is filtered out and the frequency doubled light is collimated.

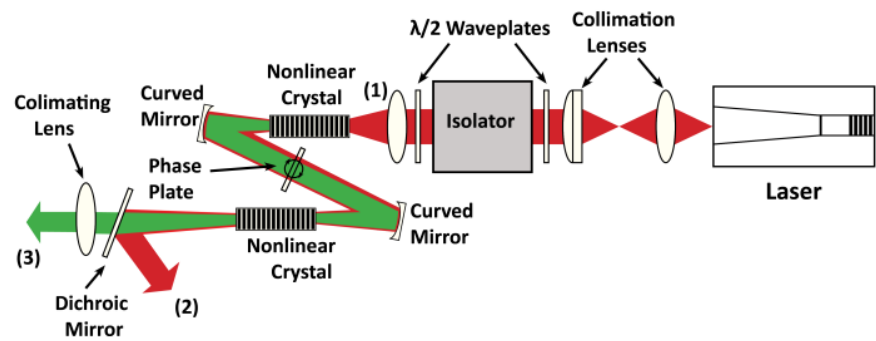

Fig. 1. Sketch of the SHG cascade laser. The fundamental IR beam is shown with red, and the SHG beam with green. Noise measurements were carried out at position (1) of the IR light before the first nonlinear crystal, at position (2) of the green SHG after passing both nonlinear crystals, and at position (3) of the IR beam after both crystals.

The generated green SHG light is used for pumping a Ti:S laser (Scientific from Femtolasers Production), which provides short pulses with a repetition rate of $90 \mathrm{MHz}$. This light source provides under mode-locked (ML) operation a broad emission spectrum with a full width at half maximum (FWHM) of about $\sim 50 \mathrm{~nm}$, indicating less than $20 \mathrm{fs}$ pulse width. Such ultrashort laser pulses are ideally suited for a range of biophotonics applications, such as various forms of imaging. In order to improve the image quality, it is of paramount importance to understand and improve the noise properties of the utilized light source.

\section{NOISE DETECTION SETUPS}

\section{A. Electrical noise}

The motivation of this work is to characterize the noise transfer through the described optical system, and the first link in the chain of noise transfer is the electrical noise of the power supplies. This section describes the setup used for measuring the electrical amplitude noise originating from the power supplies, see Fig. 2. As described in section II, the investigated diode laser consists of a RW and a TPA section. The RW section is connected to a low current power supply (Newport 6000 injecting $300 \mathrm{~mA}$ ) whereas the TPA section is connected to a high current power supply (Newport 5600 injecting $14 \mathrm{~A})$. In the test setup, each power supply is connected to resistors which simulate the voltage drop that occurs when the power supply is connected to the laser diode. The amplitude noise is then monitored using an electrical spectrum analyser (SA) which is described in the next section. As an additional component, a high current low-pass filter (ATLF20AV, Analog Technologies) can be introduced to improve the noise performance of the high current power supply. It should be noted that these power supplies (especially the high current power supply) were chosen due to the presence of signature noise properties. As will be shown, the relatively high noise level of the high current power supply makes it easier to investigate and follow the noise transfer through the entire chain.

Finally, the background measurements were performed by attaching the investigated power supplies to the noise detection setup but without turning them on. This is done to ensure that any external influence (e.g. radio-frequencies), would be considered in the measurements and not interpreted as part of the power supply noise.

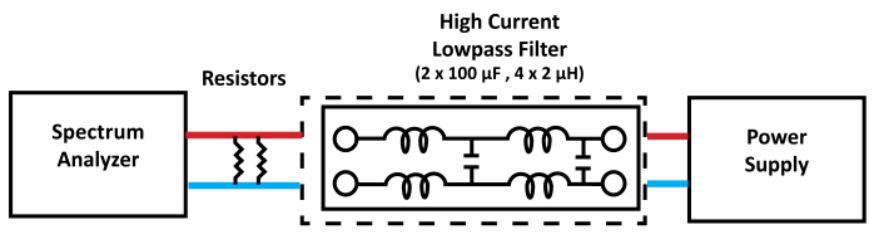

Fig. 2. Electrical noise measurement setup. The power supply is connected to resistors, which simulate the voltage drop of the diode laser. As an additional component, a low-pass filter can be introduced to filter the electrical noise of a high current power supply.

\section{B. Optical noise}

The optical intensity noise measurement setup is seen in Fig. 3. At first, the optical power of the investigated laser is adjusted using a neutral optical density filter before entering the setup. The attenuated beam is then directed at either one or both photodetectors (combined in one unit), by rotating the polarization using a $\lambda / 2$ plate, before entering a polarizing beam splitter. Each beam is focused onto the active area of its photodetector using a lens with focal length $f=3 \mathrm{~cm}$.

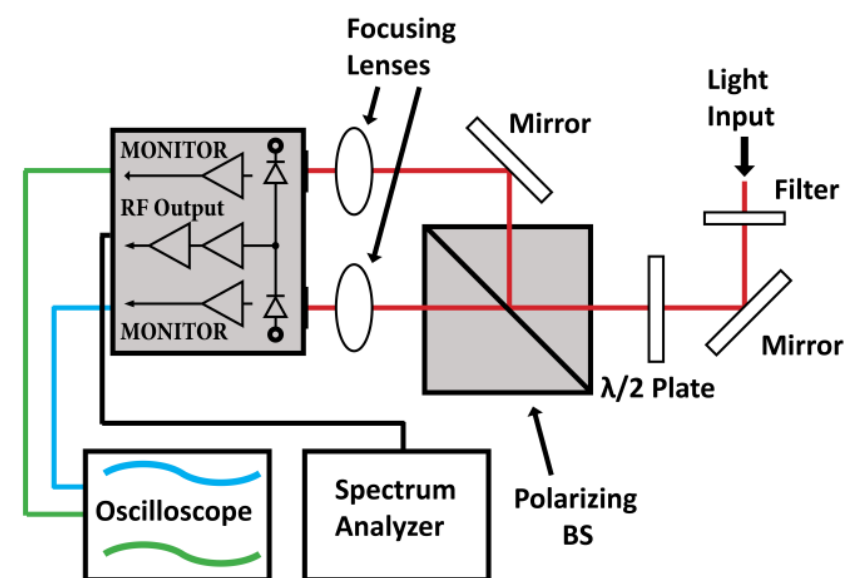

Fig. 3. Intensity noise detection setup consisting of a neutral optical density filter, a polarizing beam splitter (BS), a balanced amplified photodetector (BAP), an oscilloscope, and an electrical spectrum analyzer (SA).

The photodiode used in this work (PDB450-AC, Thorlabs) is a balanced amplified photodetector (BAP). It consists of two well-matched photodiodes combined with a so called "ultra-low noise", high speed transimpedance amplifier. The BAP generates an output voltage proportional to the difference between the photocurrents in the two photodiodes, i.e. the two optical input signals. The monitor signals of the individual photodiodes are observed using an oscilloscope, while the difference (RF output) between the two photodiodes (AC 
coupled signal) is directed to the electrical SA. Overall, the BAP corresponds to a homodyne detection system, which can provide measurements of the intensity noise and of the shot noise.

When the laser beam is incident on only one of the two photodiodes, the RF output provides the amplitude noise. However, when the beam is equally split (balanced) onto each photodiode, the RF output provides an experimental measurement of the shot noise. Note that this shot noise is not the photon shot noise of the full-power laser beam, but the electron shot noise resulting from measurement of the attenuated beam. The beam is split equally between the photodiodes by rotating the $\lambda / 2$ plate such, that the readout from the analyzer is at a minimum level, ensuring detection of the shot noise.

The photodiode is chosen mainly due to its wavelength coverage. It is silicon-based and specified for operation in the [320 - 1000] nm range, although it also works fine for wavelengths even up to about $1100 \mathrm{~nm}$ (responsivity $\sim 0.4 \mathrm{~A} / \mathrm{W}$ ). This covers the infrared (IR) pump wavelength $(1063 \mathrm{~nm})$, its SHG $(532 \mathrm{~nm})$, and the Ti:S emission spectrum [700 - 900] nm. Thus it can provide noise measurements of different wavelengths with the same device, offering easier comparison between measurements. The input optical power is adjusted so that the monitor output voltage is $\sim 1.4 \mathrm{~V}$ for each measurement. This value of $1.4 \mathrm{~V}$ is chosen as a compromise between obtaining a high signal strength and yet maintaining linearity of the detector.

The signal from the photodiode is investigated using a SA, which can measure the amplitude modulation spectrum of the photoreceiver signal. The SA used in this work (Tektronix 2753P), is capable of providing frequency scans in the range of $[100-1.8 \mathrm{G}] \mathrm{Hz}$, with average background levels between -110 and $-150 \mathrm{dBm} / \mathrm{Hz}$, which basically sets the minimum detectable signal. In this investigation, noise measurement scans were only performed up to $100 \mathrm{MHz}$ as the photodiode has a cut-off frequency at $45 \mathrm{MHz}$ at the chosen gain setting of $A_{\mathrm{g}}=1 / 2 \times 10^{4} \mathrm{~V} / \mathrm{A}$.

For characterizing the performance, sensitivity, and background of the noise measurement setup itself, measurements under four different conditions are compared to each other, illustrating the sources of the various background contributions and noise signatures:

A. Measurement with SA input connected only to a $50 \Omega$ terminator.

B. Measurement with SA input connected to the BAP. The BAP is powered and set to a gain of $10^{4} \mathrm{~V} / \mathrm{A}$, but no laser light is incident on its photodiodes.

C. As (B), but with laser light incident on both photodiodes, with equal powers.

D. As (B), but with laser light incident on only one of the photodiodes.

Measurement condition $\mathbf{D}$ corresponds to a measurement of the laser beam amplitude noise. Measurements $\mathbf{A}, \mathbf{B}$, and $\mathbf{C}$ identify the measurement limitations due to the background.

\section{Reference measurement}

In this section, a reference measurement is performed on a commercial solid-state laser (Coherent VERDI V5). The specified relative intensity noise (RIN) of this laser is $<0.02 \%$ rms integrated over the interval [10 - $1 \mathrm{M}] \mathrm{Hz}$, and measurements describing its noise properties have previously been reported [17]. This characterization was performed by measuring the noise floor of the SA (A), the noise floor of the photodiode when connected to the SA (B), the balanced measurement $(\mathbf{C})$ from which a measurement of the shot noise can be extracted, and finally the amplitude noise (D) of the solid-state laser, see Fig. 4.

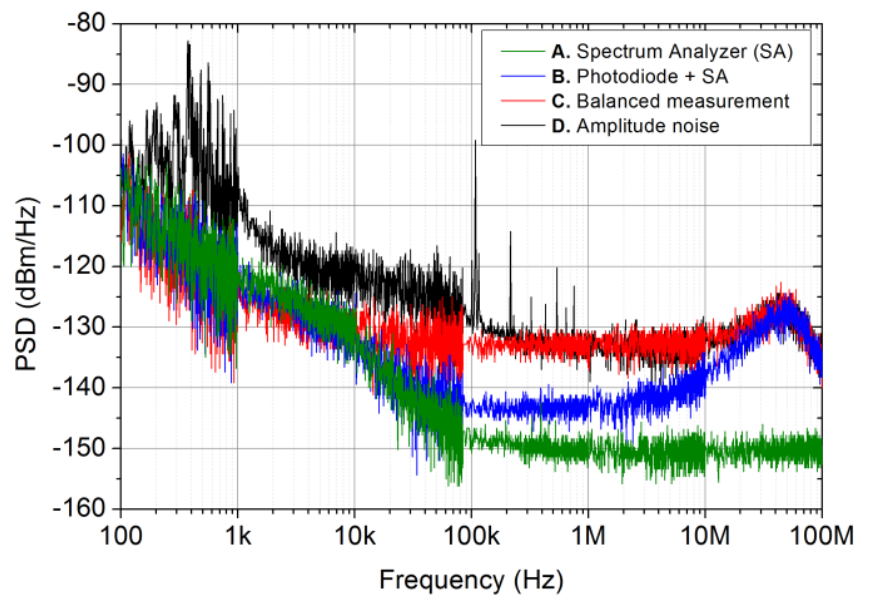

Fig. 4. Power Spectral Density (PSD) of the measurements of the reference laser, taken in configurations $\mathbf{A}$ (green), $\mathbf{B}$ (blue), $\mathbf{C}$ (red), and $\mathbf{D}$ (black). The areas where the black curve is above the red curve corresponds to measurements of the actual laser amplitude noise.

It is seen that the SA has the lowest background at higher frequencies followed by the photodiode noise floor. The amplitude noise of the laser has the highest noise level, until it reaches the shot noise level, which is about $10 \mathrm{~dB}$ above the photodiode background. However, at lower frequencies (up to $10 \mathrm{kHz}$ ) the SA-, photodiode, and the balanced measurement overlap and are not clearly separated. Nevertheless, the amplitude noise level can still be observed in this frequency range, rising $\sim 10 \mathrm{~dB}$ above the other levels. At $45 \mathrm{MHz}$, an increase in the background level is seen, which corresponds to the cut-off frequency of the photodiode amplifier described earlier. This measurement was done by stitching 6 frequency scans together at $1 \mathrm{kHz}, 10 \mathrm{kHz}, 85 \mathrm{kHz}, 1 \mathrm{MHz}$, and at $10 \mathrm{MHz}$. The individual scans were performed with different measurement bandwidths, hence the changes in the vertical width of the individual curves at the above frequencies.

As mentioned, the solid state laser was chosen in order to compare its results with published results. By comparing the obtained amplitude noise in Fig. 4 with the results published in [17], similar noise structures are observed. In the [100 $1 \mathrm{k}] \mathrm{Hz}$ region, similar multiples of peaks are observed, however a certain noise peak at $\sim 3 \mathrm{kHz}$ is not observed in our measurement. Nevertheless, in the frequency range [100k $1 \mathrm{M}] \mathrm{Hz}$, similar peaks are observed, corresponding to harmonics of the main peak at $108 \mathrm{kHz}$. Finally, in the range [1 - 10] MHz, the amplitude noise goes toward the shot noise 
limit, in accordance with the previously published results.

The unit of the power spectral density (PSD) as seen in Fig. 4 is converted from $\mathrm{dBm} / \mathrm{Hz}$ to $\mathrm{dBc} / \mathrm{Hz}$ using the relation $S_{p}(f)[\mathrm{dBc} / \mathrm{Hz}]=$

$$
S_{p}(f)[\mathrm{dBm} / \mathrm{Hz}]-10 \log _{10}\left(\frac{U^{2}}{R_{T}} 1 \mathrm{~mW}^{-1}\right),
$$

where $U$ is the DC voltage that would have entered the spectrum analyzer termination $R_{\mathrm{T}}$ had the BAP not been AC coupled, and the factor of $1 \mathrm{~mW}^{-1}$ provides a unit-less expression inside the parenthesis. The DC voltage is given by $U=R(\lambda) P_{O P} A_{g}$,

where $R(\lambda)$ is the wavelength responsivity [A/W], which takes into account the quantum efficiency of the detector. $A_{\mathrm{g}}$ is the transimpedance gain [V/A], and $P_{\mathrm{OP}}$ is the average optical power at the detector [W]. The photodiode amplifier has a gain of $A_{\mathrm{g}}=1 / 2 \times 10^{4} \mathrm{~V} / \mathrm{A}$ through a $50 \Omega$ termination. This conversion makes it possible to normalize the PSD to the carrier (DC level), and hence compare different noise measurements performed with different wavelengths/optical powers. The RIN can then be obtained by integrating the normalized PSD over an interval $\left[f_{1}-f_{2}\right]$ of noise frequencies to obtain a root mean square (rms) value of the RIN:

$$
R I N=\sqrt{\int_{f_{1}}^{f_{2}} S_{p}(f) d f},
$$

which is often specified in percent [18]. To show the significance/contributions of the different noise, Fig. 5 shows the integrated mean squared RIN, i.e., $\mathrm{RIN}^{2}$, of the amplitude noise and shot noise, starting at $1 \mathrm{MHz}$ and going down to $100 \mathrm{~Hz}$. The corresponding RIN value is $0.0056 \% \mathrm{rms}$, in accordance with the value of $<0.02 \% \mathrm{rms}$, specified by the manufacturer. However, it is important to note that the manufacturer specifies this value for integration from [10 $100 \mathrm{M}] \mathrm{Hz}$, whereas the integration in Fig. 5 is only performed up to $1 \mathrm{MHz}$, since it is clear that the amplitude noise goes toward the shot noise limit around this frequency. By further integration, additional contribution from the amplifiers frequency cut-off would be added and thus provide a wrong RIN value.

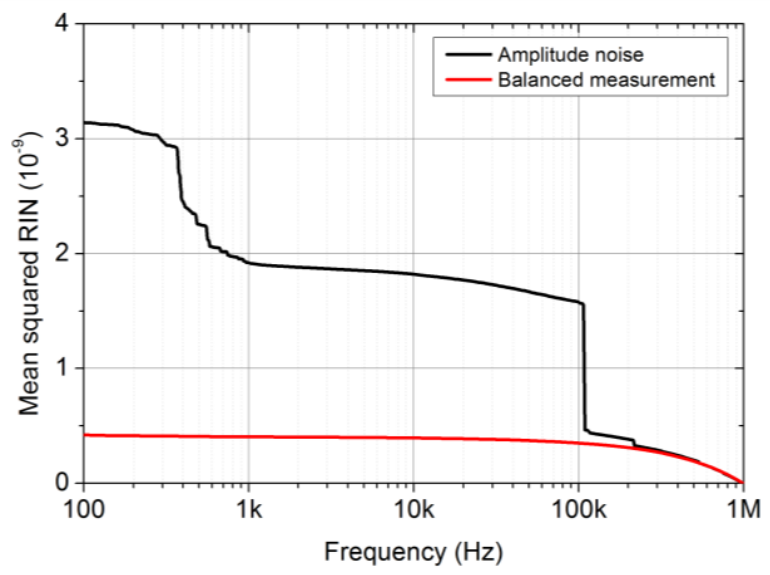

Fig. 5. Mean squared RIN calculation by integration of the $S_{\mathrm{p}}(f)$ and of the shot noise $S_{\mathrm{p}, \mathrm{SN}}(f)$ from $1 \mathrm{MHz}$ down to $100 \mathrm{~Hz}$.
Finally, Fig. 5 also shows which frequencies of the amplitude noise (Fig. 4) contribute the most to the RIN; the peaks at $[300-1 \mathrm{k}] \mathrm{Hz}$ and at $[100-300] \mathrm{kHz}$. The reference measurement is thus in good agreement with the literature and with the specifications of the reference laser.

\section{RESULTS}

In this section the results of the noise measurements of the optical system are presented. This study contains both electrical and optical noise measurements describing the noise transfer from the power supplies into the IR beam, into its SHG, and finally into the Ti:S laser.

\section{A. Electrical noise}

The first link in the chain of noise transfer is to investigate the electrical noise properties of the power supplies. As described earlier, the investigated tapered diode laser consists of a RW and a TPA section, each with separate injection current contacts.

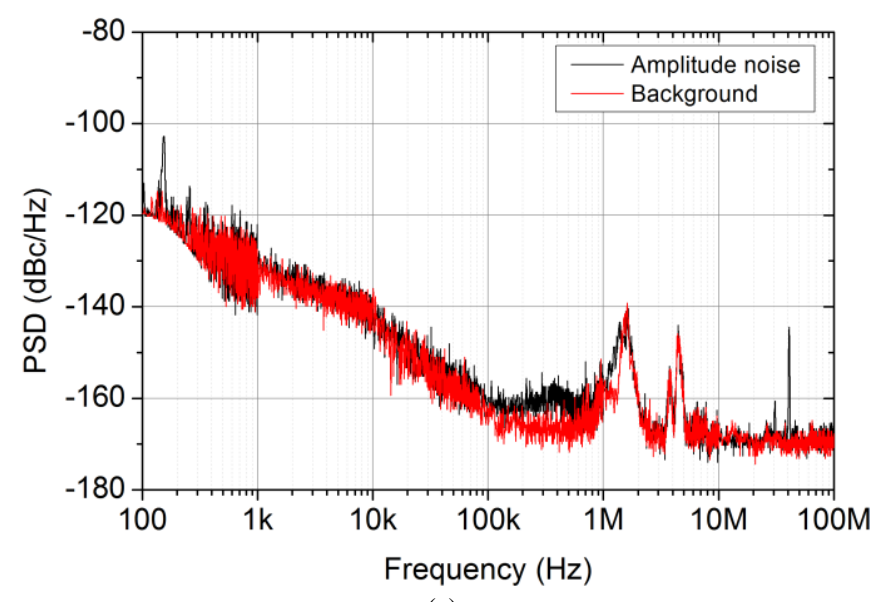

(a)

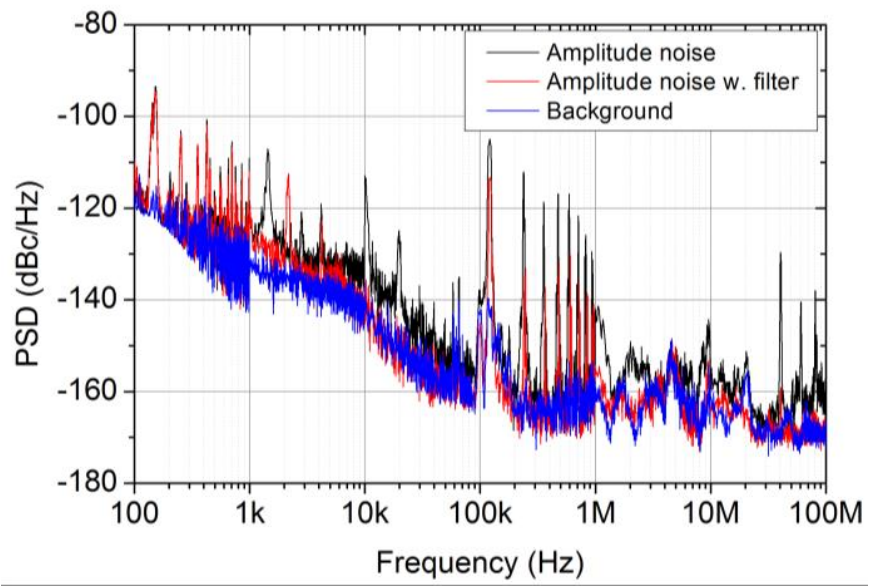

(b)

Fig. 6. Electrical amplitude noise and background measurements of (a) the low current power supply operated at $300 \mathrm{~mA}$ and (b) high current power supply operated at $14 \mathrm{~A}$, with and without the low-pass filter.

The RW is connected to a low current power supply (Newport 6000) operated at $300 \mathrm{~mA}$. The electrical amplitude noise measurement of this power supply is seen in Fig. 6(a), however the noise was too low to be detected by our setup. As seen, the obtained measurements were very similar to the 
background measurement with small deviations. To enable easy comparison of the background to the amplitude noise measurement, the DC value of the latter was also used to convert the former to a $\mathrm{dBc} / \mathrm{Hz}$ scale.

The TPA section was connected to a high current power supply (Newport 5600) operated at $14 \mathrm{~A}$, which in addition could be operated with a high current low-pass filter, see Section III(A). The electrical noise measurements of this power supply, with and without the low-pass filter are shown in Fig. 6(b). Multiple peaks are seen over the detection frequency range with the most evident peaks in the $[100 \mathrm{k}-$ $2 \mathrm{M}] \mathrm{Hz}$ range. The effect of the low-pass filter is also seen, with a reduction of about $10 \mathrm{~dB}$ in the $\mathrm{kHz}$ region. The amplitude of the peaks between $100 \mathrm{kHz}$ and $2 \mathrm{MHz}$ were also reduced, while the noise level in the $[100-1 \mathrm{k}] \mathrm{Hz}$ region was not affected by the introduction of the low-pass filter, which is in good agreement with the specifications of the filter. Nonetheless, it is important to highlight the difference between the impedance of the applied load resistor and the more complex impedance of the actual diode laser.

Finally, note that this power supply was not chosen due to its noise performance, where in comparison batteries are known to offer low noise. In fact, the noise features of this power supply are necessary to follow the noise development at the different stages, which is the aim of this study.

\section{B. IR laser noise}

The next link in the noise chain is to consider the electrooptical noise coupling by studying the IR radiation from the tapered diode laser, when operated with the before mentioned power supplies. Fig. 7 shows the optical amplitude noise of the tapered diode laser operated with and without the low-pass filter (measured at (1) in Fig. 1).

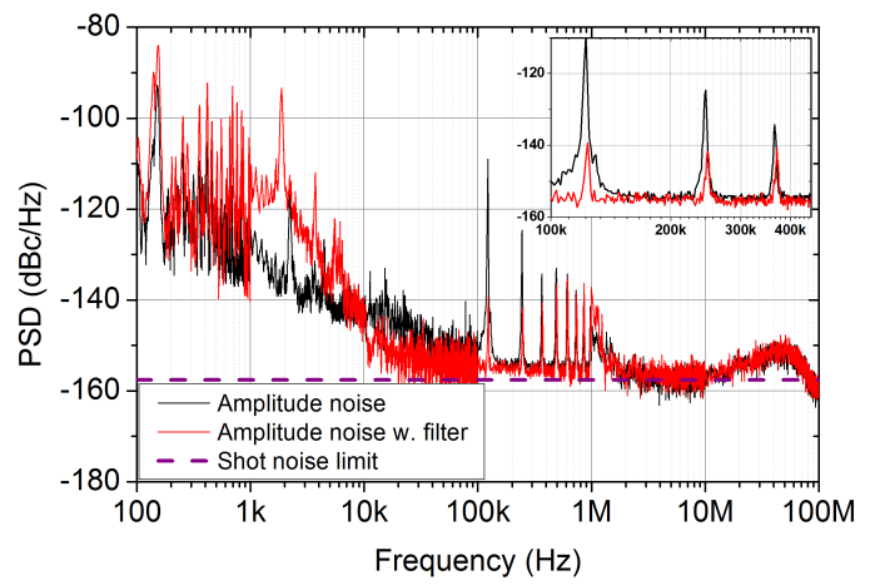

Fig. 7. Amplitude noise of the tapered diode laser, together with the fundamental shot noise limit. The laser was operated with and without the low-pass filter connected to the high current power supply. Inset: Zoom of the "power supply" peaks.

These measurements show noise structures very similar to those seen in the high current power supply measurements, see Fig. 6(b). The main peaks in the $[100 \mathrm{k}-2 \mathrm{M}] \mathrm{Hz}$ region are clearly seen, and even some of the peaks in the $[1-10] \mathrm{kHz}$ region are transferred from the high current power supply. However, at higher frequencies ( $>2 \mathrm{MHz}$ ), the electrical noise structures are no longer seen in the IR noise measurements, where the optical noise level goes toward the shot noise limit at $\sim 2 \mathrm{MHz}$ at a level of $-158 \mathrm{dBc} / \mathrm{Hz}$. The IR light has a RIN value of $0.016 \%$ rms integrated between $[10-1 \mathrm{M}] \mathrm{Hz}$.

The effect of introducing the high current low-pass filter is also evident in the IR noise measurements, as the noise level in the range $[10 \mathrm{k}-1 \mathrm{M}] \mathrm{Hz}$ is reduced. Surprisingly, the optical noise seems to have increased in the range [1 $10] \mathrm{kHz}$, an effect which was not observed for the electrical noise, but the overall noise level is improved by the introduction of the low-pass filter.

\section{SHG laser noise}

After measuring the amplitude noise of the infrared laser emission, the next stage is to investigate the noise of the light generated by the SHG process. In this section, where the focus will be on the intensity noise of the different SHG stages, the effect of introducing the low-pass filter will not be shown for brevity, although a similar filtering effect occur. As described earlier, the investigated laser system consists of a cascade of two nonlinear crystals, in which the green laser light is generated. Fig. 8 shows noise measurements of the different stages of the SHG process (measured at (2) in Fig. 1). This includes the case where only one of the two (first or second) nonlinear crystals fulfils the phase-matching condition, as well as the case where both crystals are phase matched. Experimentally, this was done by changing the temperature set points of the individual ovens in which the nonlinear crystals were placed.

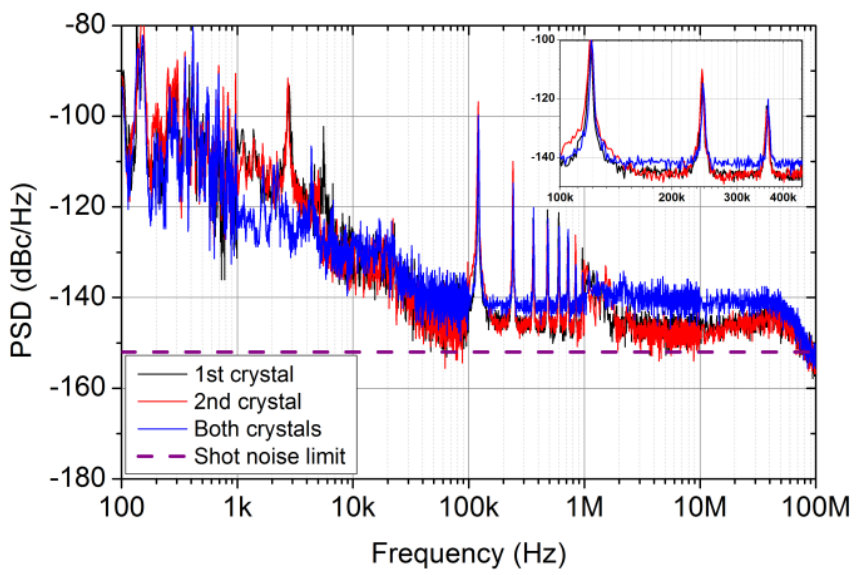

Fig. 8. Amplitude noise of the SHG light for the cases where the light is generated from only the 1 st (black), only the 2 nd (red) or from both nonlinear crystals (blue). The shot noise limit (purple) is also shown for the SHG light. Inset: Zoom of the power supply peaks.

First of all, it is seen that the SHG light and the fundamental IR light have similar noise structures, originating from the high current power supply. It also seems to indicate that the SHG light is no longer shot noise limited in the detected range, unlike the IR noise in Fig. 7. A similar behaviour of increased SHG noise relative to the IR noise has been reported in [19]. In the frequency range $[100-1 \mathrm{M}] \mathrm{Hz}$, the cascaded SHG light has a RIN value of $0.062 \%$ rms.

In addition, the noise level seems to depend on whether one or both crystals are phase matched. At higher frequencies, an almost constant increase (white noise) in the noise level is observed, which is higher in the case of having both crystals 
phase matched than in the case of only one single phase matched crystal, as seen in the inset of Fig. 8. At lower frequencies however, there is no clear differentiation between the amplitude noises from one or two phase matched crystals.

\section{IR noise and the SHG process}

In this section, similar noise investigations of the SHG process were carried out, but instead of measuring the noise of the SHG light, the noise of the residual IR beam was measured (at position (3) in Fig. 1), after passing no phase matched crystals, one phase matched crystal (1st or 2 nd), or after passing both phase matched crystals. The measurements are shown in Fig. 9. A similar trend of increased intensity noise in the case of two phase matched crystals is seen for the IR noise, as in the SHG noise measurement. This increase seems to be highest at lower frequencies and drops off in a $1 / f$ manner (pink noise), while at higher frequencies, a constant increase in the noise level (white noise) can barely be distinguished.

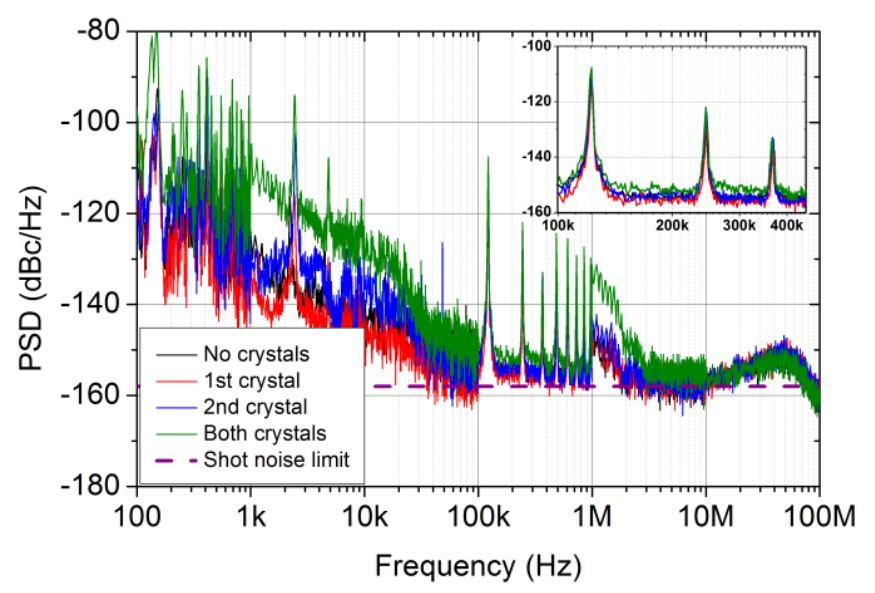

Fig. 9. Amplitude noise of the IR beam after passing no phase matched crystals (black), after passing the 1 st (red), the 2 nd (blue), or both nonlinear crystals (green) while being phase matched (generating SHG light). The corresponding shot noise limit is also shown. Inset: Zoom of the power supply peaks.

\section{E. Ti:S laser noise}

The final step of the noise transfer is the Ti:S laser noise under continuous wave (CW) and ML operation, see Fig. 10, which also includes the corresponding noise of the pump source (SHG cascade). By comparing the noise of the pump source and the Ti:S, it is once again evident that the main noise features of the power supply are transferred through the optical system and are observed in the Ti:S noise.

That said, a new 'plateau' from $30 \mathrm{kHz}$ up to $500 \mathrm{kHz}$ is seen, which presumably originates from the relaxation oscillation frequency (ROF) of the Ti:S [17]. Finally, a new peak at $90 \mathrm{MHz}$ is observed under ML operation, which corresponds to the repetition rate of the laser and is thus not actual noise. Overall the noise level is higher under ML operation compared to the $\mathrm{CW}$ case. Between $[100-1 \mathrm{M}] \mathrm{Hz}$, the Ti:S has a RIN value of $0.21 \%$ rms under $\mathrm{CW}$ operation, while it has $0.24 \%$ rms under ML operation.

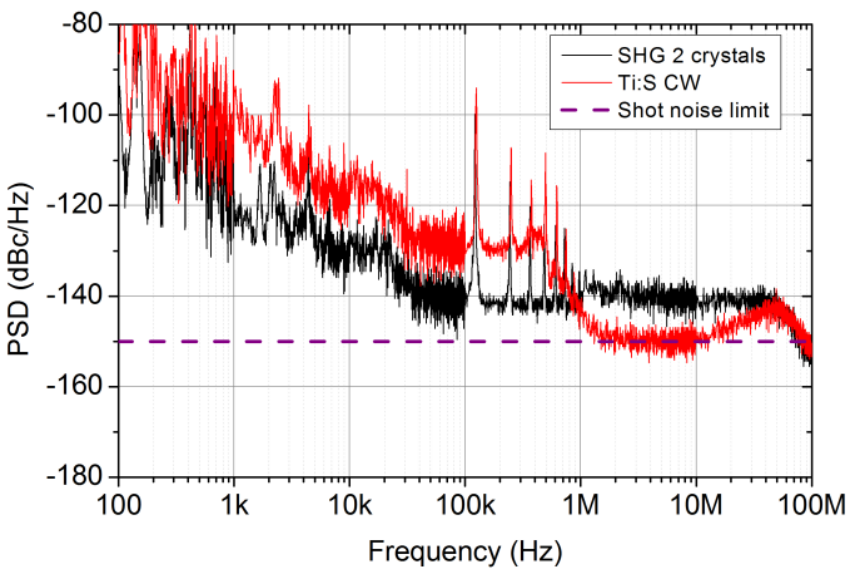

(a)

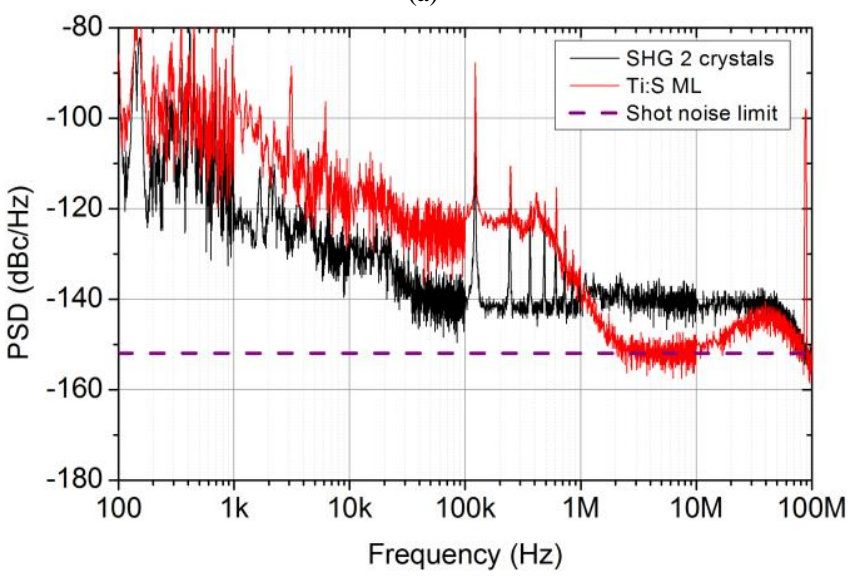

(b)

Fig. 10. Amplitude noise of the Ti:S laser (red) under CW (a) and ML (b) operation. The figure also includes the corresponding intensity noise of the pump source (black): SHG light generated from the cascade system. The shot noise (purple) indicated is that of the Ti:S emission.

\section{DISCUSSION}

The different noise stages of this investigation are summarized in Fig. 11. The electrical noise of the (high current) laser driver is transferred into the optical (IR) noise of the laser, which in our measurement became shot noise limited at around $2 \mathrm{MHz}$. However, this does not imply that the electrical noise spectra above $2 \mathrm{MHz}$ are not transferred into the optical noise, as such structures will still exist in the optical noise. In fact, these structures would be resolved if the noise measurement could be performed with higher optical input powers, where the relative shot noise of the measurement would be lower. Additionally, the broad background peak at $45 \mathrm{MHz}$, arising from the cut-off frequency of the BAP's transimpedance amplifier, conceals noise signatures with a lower spectral power, e.g. the electrical noise peak at $40 \mathrm{MHz}$, see Fig. 11 .

Overall, the observed behaviour of the SHG noise follows that of the IR noise. The SHG light has an increased noise level compared to the fundamental light, and the same applies to the remaining IR light after passing the SHG process. The increase in IR noise is to be expected as the powers of the two electromagnetic fields (IR and SHG) are correlated. Since the frequency conversion process is quadratic in the input optical 
power, one would expect the relative SHG amplitude noise to be a factor of 2 (6 dB electrical) larger than that of the input IR, similar to the increase in amplitude noise observed in [19]. That said, the additional white noise in the case of two phase matched crystals is somewhat surprising and its origin is not clear.

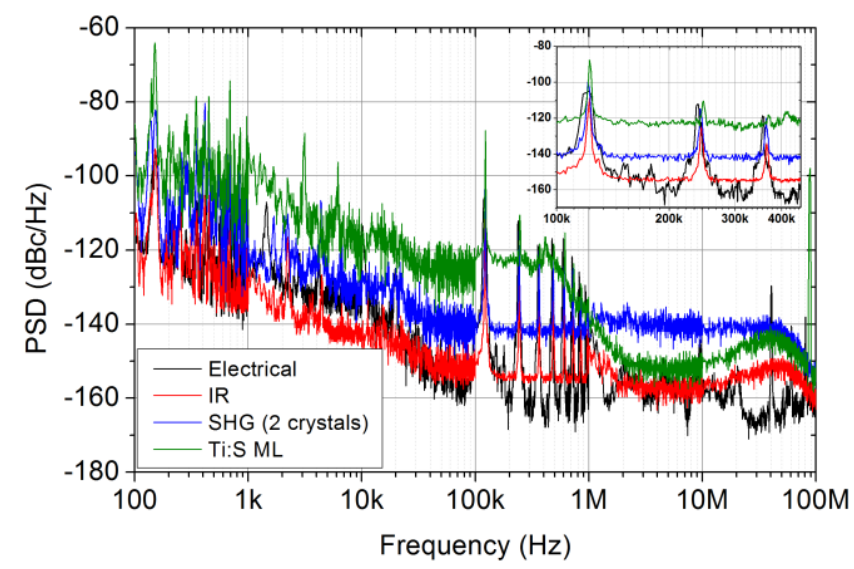

Fig. 11. Amplitude noise of the different stages in the system; the electrical noise of the high current supply (black), the IR light (red), the SHG light generated from both nonlinear crystals (blue), and the corresponding amplitude noise of the Ti:S laser under ML operation (green). Inset: zoom of the power supply peaks.

One might think that the generated SHG light from the second crystal is incoherent with that generated from the first crystal, and that the noise contributions from the two stages are thus simply added. This is however not the case since a cascaded system increases the SHG power by constructive inference, which is based on a coherent interaction between two electromagnetic fields [12]. In addition, Fig. 12 in the Appendix shows the amplitude noise of $(\sim 1.1 \mathrm{~W})$ SHG light, when generated from only the first nonlinear crystal and when generated from both (cascaded) crystals. This measurement indicates that the increased noise is due to an interaction between the two crystals, and not dependent on the generated SHG power. The exact reason for the increased noise in the cascaded system relative to the single crystal is from this investigation unclear.

The Ti:S showed up to $500 \mathrm{kHz}$ a $10-20 \mathrm{~dB}$ increased noise level, relative to the SHG noise. According to the complex noise transfer theory [21],[22], a linear transfer of the pump source noise is expected below the ROF, followed by a second-order pole with $-40 \mathrm{~dB} /$ decade rolloff at frequencies above the ROF. From the Ti:S noise measurements, one can deduce that the cut-off frequency of the complex noise function is around $500 \mathrm{kHz}$. This is in good agreement with the upper state lifetime of $3.2 \mu \mathrm{s}$ in the $\mathrm{Ti}: \mathrm{S}$ and also agrees well with [21]. A cut-off frequency in the $\mathrm{kHz}$ range is ideal for diode based pump lasers, which have ROF in the $\mathrm{GHz}$ range. This would provide filtering of all noise contributions of the pump laser above the ROF of the Ti:S.

Finally, it seems that the main part of the Ti:S laser noise originates from the laser itself. The ROF can be shifted toward higher frequencies by increasing the output power of the laser pump [18], which is also confirmed in Fig. 13 in the Appendix, that shows the noise of the Ti:S when pumped with the solid state laser (Coherent VERDI V5) with different pump powers. As the pump power increases, a general decrease of the Ti:S noise at low frequencies is also observed. As described in Section II, the Ti:S was pumped with $2.8 \mathrm{~W}$ of SHG light generated from the cascaded system. This suggests that by increasing the pump power of the Ti:S, the ROF can be increased past the cut-off frequency resulting in an overall improved noise performance.

\section{CONCLUSION}

In this work, we have studied the noise performance and transfer through a laser system suitable for among other biomedical imaging applications. This system consists of a DBR tapered diode laser, a cascade of two nonlinear crystals for SHG, and a Ti:S laser configured to be pumped by the SHG light. This investigation includes electrical noise measurements of the power supplies as well as the optical noise of the different stages. The RIN values of these stages in the range $100 \mathrm{~Hz}-1 \mathrm{MHz}$ are summarized in Table 1 .

TABLE I

RIN VALUES AT THE DIFFERENT STAGES

\begin{tabular}{ccccc}
\hline \hline & Electronic & IR & SHG & Ti:S ML \\
\hline RIN [\% rms] & 0.051 & 0.016 & 0.062 & 0.24 \\
\hline \hline
\end{tabular}

Through this study, we have identified that the electrical noise of the low current power supply was not apparent with our noise detection setup and its effect was not noticeable in the optical noise. The main source behind the optical noise is the electrical noise of the high current power supply, which injects current into the tapered section of the laser.

This is evident as the main noise peaks in the electrical noise were transferred throughout the entire optical system. The effect of introducing a high current low-pass filter was also clear, and not only the electrical but also the optical noise was reduced. This indicates that by simply using power supplies with low noise, introducing multiple electrical filters, or a combination of both could offer an easy and efficient method to improve the overall optical noise performance of a system.

While the optical noise of the tapered diode laser was below the detection shot noise limit at frequencies above $2 \mathrm{MHz}$, the SHG light had an increased noise level. This was most clear in the case of the cascaded system with white noise rising almost $3 \mathrm{~dB}$ above shot noise in the detected frequency range. Similarly, the noise level of the remaining IR light was increased in a similar fashion to the SHG noise.

The final link in the noise transfer chain, from the SHG pump source to the Ti:S, showed a cut-off frequency around $500 \mathrm{kHz}$, roughly equal to the ROF of the Ti:S cavity. In accordance with theory, noise features above the cut-off frequency were strongly damped. Despite the generally higher noise in the Ti:S laser, its relative noise nevertheless dropped below that of the SHG light at frequencies above $1 \mathrm{MHz}$. In addition, it seems that the majority of the Ti:S noise originates from the laser itself. However, increasing the output power of 
the pump laser, results in an overall improved noise performance at both high and low frequencies.

While the ideal case would be pumping the Ti:S with a low noise pump laser, the observed results suggests that pump laser noise features above the cut-off frequency are tolerable, as such features would be filtered away. This makes diode based pump sources excellent as their ROF lies in the $\mathrm{GHz}$ range. In conclusion, this study serves as an investigation of the noise transfer through the described setup. It is based on a power supply with relatively high and distinct noise features, which makes it possible to follow the noise transfer at the different stages.

\section{APPENDIX}

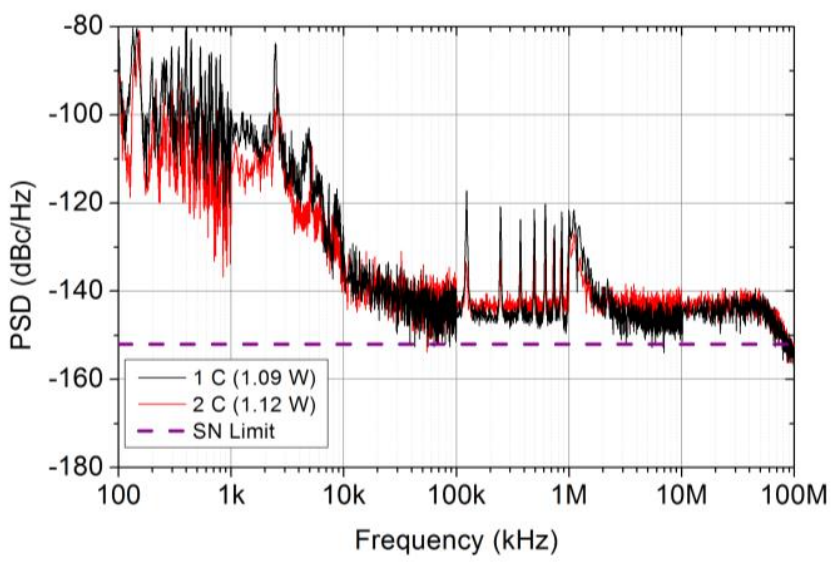

Fig. 12. Amplitude noise of the SHG light, when $\sim 1.1 \mathrm{~W}$ of green light is generated from either 1 ( $1 \mathrm{C}$ black) or from $2(2 \mathrm{C}$ red $)$ phase matched crystals. The corresponding shot noise limit (purple) is also shown.

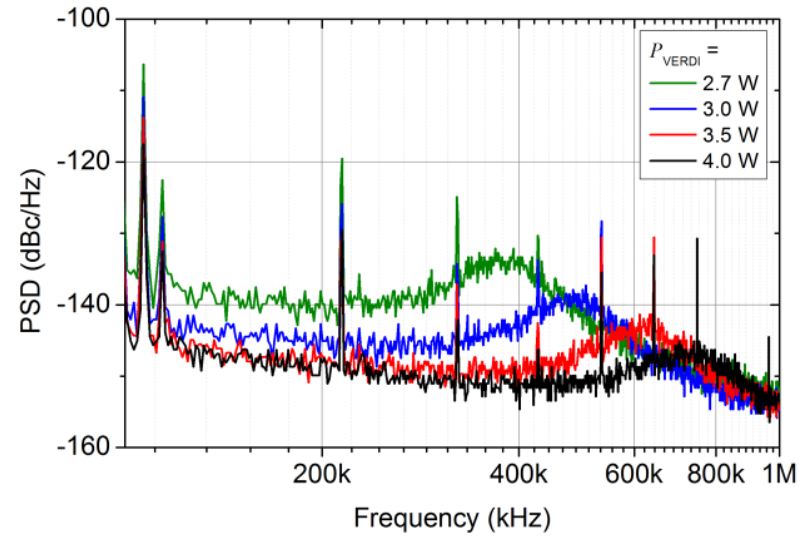

Fig. 13. Amplitude noise of the Ti:S pumped by the VERDI V5, at different pump powers. The ROF is shifted toward higher frequencies at higher pump powers. The amplitude noise for all four measurements is shot noise limited at $1 \mathrm{MHz}$. This zoom is made to emphasis on the relationship between the ROF and the green pump power.

\section{REFERENCES}

[1] Y. Arakawa, K. Vahala, and A. Yariv "Quantum noise and dynamics in quantum well and quantum wire lasers," Appl. Phys. Lett., vol. 45, issue 9, pp. 950-952, (1984).

[2] D. J. Morgan, and M. J. Adams "Quantum noise in semiconductor lasers," phys. stat. sol. (a), vol. 11, issue 1, pp. 243-253, (1972).

[3] Y. Yamamoto "AM and FM Quantum Noise in Semiconductor LasersPart I: Theoretical Analysis," IEEE J. Quantum Electron., vol. 19, issue 1, pp. 34-46 (1983).
[4] T. A. B. Kennedy, and D. F. Walls "Amplitude noise reduction in atomic and semiconductor lasers," Phys. Rev. A, vol. 40, issue. 11, pp. 6366-6373, (1989).

[5] D. Eliyahu, D. Seidel, and L. Maleki "RF Amplitude and Phase-Noise Reduction of an Optical Link and an Opto-Electronic Oscillator," IEEE Trans. Microw. Theory Techn., vol. 56, issue 2, pp.449-456, (2008).

[6] J. Kitching, R. Boyd, A. Yariv, and Y. Shevy "Amplitude noise reduction in semiconductor lasers with weak, dispersive optical feedback," Opt. Lett., vol. 19, issue 17, pp. 1331-1333 (1994).

[7] W. J. Brown, S. Kim, and A. Wax "Noise characterization of supercontinuum sources for low-coherence interferometry applications," J. Opt. Soc. Am. A, vol. 31, issue 12, pp. 2703-2710, (2014).

[8] S. Bourquin, A. D. Aguirre, I. Hartl, P. Hsiung, T. H. Ko, J. G. Fujimoto, T. A. Birks, W. J. Wadsworth, U. Bünting, and D. Kopf "Ultrahigh resolution real time OCT imaging using a compact femtosecond Nd:Glass laser and nonlinear fiber," Opt. Express, vol. 11, issue 24, pp. 3290-3297, (2003).

[9] M. Jurna, J.P. Korterik, C. Otto, and H.L. Offerhaus "Shot noise limited heterodyne detection of CARS signals," Opt. Express, vol. 15, issue 23, pp. 15207-15213, (2007).

[10] B. Sumpf, K.-H. Hasler, P. Adamiec, F. Bugge, F. Dittmar, J. Fricke, H. Wenzel, M. Zorn, G. Erbert, and G. Tränkle "High-Brightness Quantum Well Tapered Lasers" IEEE J. Sel. Top. Quantum Electron., vol. 15, issue 3, pp. 1009-1020, (2009).

[11] O. B. Jensen, A. K. Hansen, A. Müller, B. Sumpf, A. Unterhuber, W. Drexler, P. M. Petersen, and P. E. Andersen "Power Scaling of Nonlinear Frequency Converted Tapered Diode Lasers for Biophotonics," IEEE J. Sel. Top. Quantum Electron., vol. 20, issue 2, pp. 307-321, (2014).

[12] A. K. Hansen, M. Tawfieq, O. B. Jensen, P. E. Andersen, B. Sumpf, G. Erbert, and P. M. Petersen "Concept for power scaling second harmonic generation using a cascade of nonlinear crystals," Opt. Express, vol. 23, issue 12, pp. 15921-15934, (2015).

[13] A. Jechow, R. Menzel, K. Paschke, and G. Erbert "Blue-green light generation using high brilliance edge emitting diode lasers," Laser Photon. Rev., vol. 4, issue 5, pp. 633-655, (2010).

[14] A. Unterhuber, B. Považay, A. Müller, O. B. Jensen, M. Duelk, T. Le, P. M. Petersen, C. Velez, M. Esmaeelpour, P. E. Andersen, and W. Drexler "Simultaneous dual wavelength eye-tracked ultrahigh resolution retinal and choroidal optical coherence tomography," Opt. Lett., vol. 38, issue 21, pp.4312-4315, (2013).

[15] K. König, P. E. Andersen, T. Le, and H. G. Breunig "Multiphoton imaging with a novel compact diode-pumped Ti:sapphire oscillator," Microsc. Res. Tech., vol. 78, issue 12, pp. 1154-1158, (2015).

[16] A. Müller, S. Marschall, O. B. Jensen, J. Fricke, H. Wenzel, B. Sumpf, and P. E. Andersen "Diode laser based light sources for biomedical applications, " Laser \& Photon. Rev., vol. 7, issue 5, pp. 605-627, (2013).

[17] R.P. Scott, C. Langrock, B.H. Kolner "High-dynamic-range laser amplitude and phase noise measurement techniques," IEEE J. Sel. Topics Quantum Electron., vol. 7, issue 4, pp. 641-655, (2001).

[18] G. P. Agrawal, and N. K. Dutta, "Noise Characteristics," in Semiconductor Lasers, 2nd version, Springer US, 1993, ch. 6, sec. 6.5.2, pp. 261-269.

[19] Y. Wang, J. Fonseca-Campos, W.-G. Liang, C.-Q. Xu, and I. VargasBaca, "Noise Analysis of Second-Harmonic Generation in Undoped and MgO-Doped Periodically Poled Lithium Niobate," Adv. Optoelectron., Art. ID 428971, (2008).

[20] Y. Yamamoto, T. Mukai, and S. Saito "Quantum phase noise and linewidth of a semiconductor laser," Electron. Lett., vol. 17, issue 9, pp. 327-329, (1981).

[21] T. D. Mulder, R. P. Scott, and B. H. Kolner "Amplitude and envelope phase noise of a modelocked laser predicted from its noise transfer function and the pump noise power spectrum," Opt. Express, vol. 16, issue 18, pp. 14186-14191, (2008).

[22] C. C. Harb, T. C. Ralph, E. H. Huntington, D. E. McClelland, H.-A. Bachor, and I. Freitag "Intensity-noise dependence of Nd:YAG lasers on their diode-laser pump source," J. Opt. Soc. Am. B, vol. 14, issue 11, pp. 2936-2945, (1997). 


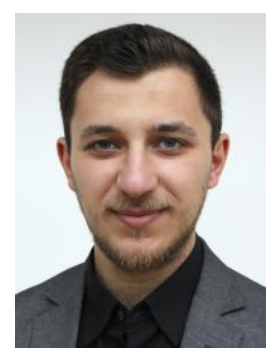

Mahmoud Tawfieq received the M.Sc. degree in physics and nanotechnology from the Technical University of Denmark, Lyngby, Denmark in 2015. From 2014 to 2015, he was working as a Production Technician at Norlase ApS. Since then, he has joined the FerdinandBraun-Institute, Berlin, Germany, where he has been working toward the Ph.D. degree. His research work is focused on developing tunable high-power MOPA systems, to serve as pump sources in upconversion systems for mid IR detection and imaging.

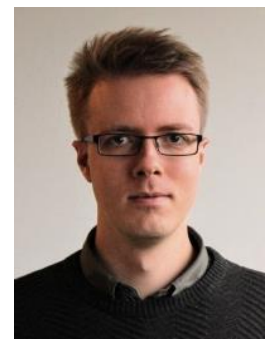

Anders Kragh Hansen received the M.Sc. and Ph.D. degrees in physics in 2010 and 2013, respectively, working on laser cooling of molecular ions in the Ion Trap Group at Aarhus University. In 2013, he joined DTU Fotonik at the Technical University of Denmark as a postdoc, where he is currently a researcher working on laser systems for biophotonics. He has a special interest in nonlinear frequency conversion, laser diodes and phase manipulation of light.

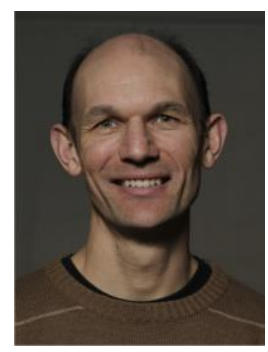

Ole Bjarlin Jensen received the M.Sc. degree in applied physics from the Technical University of Denmark (DTU), Lyngby, Denmark, in 1999 and the Ph.D. degree in optics from DTU in 2002. From 2002 to 2003, he was Development Engineer at ASAH Medicos A/S. In 2004, he joined the Ris $\varnothing$ National Laboratory as Postdoctoral Researcher and in 2007 the Technical University of Denmark as a Researcher. In 2011, he became Senior Researcher at the Department of Photonics Engineering, DTU Fotonik. His current research interests include diode lasers, solid state lasers, and nonlinear frequency conversion as well as biomedical applications of laser systems.

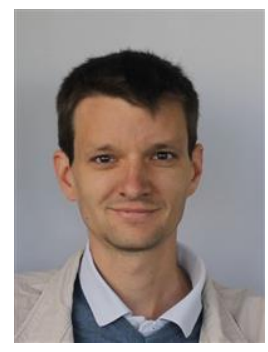

Dominik Marti received the M.Sc. and Ph.D. degrees in physics in 2006 and 2011, respectively, working on twophoton microscopy and pulsed laser micro-manipulation at the University of Bern. He did a postdoc in ultrashort pulsed laser applications in biology at the same university for 3 years, before joining DTU Fotonik in 2014, where he researches laser-based multimodal endoscopy for in vivo cancer detection.

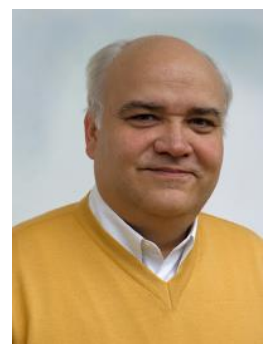

Bernd Sumpf received the Diploma degree in physics in 1981 and the Ph.D. degree in 1987 from the HumboldtUniversitat zu Berlin for his work on lead salt diode lasers for spectroscopic applications. From 1993 to 1997, he worked at the Technische Universitat Berlin on high-resolution spectroscopy and nonlinear optics and received the postdoctoral lecture qualification in 1997. Since 2000, he has been at the Ferdinand-Braun-Institut, Berlin, Germany. His current research interests include high-brightness diode lasers and diode lasers for sensory applications and RAMAN spectroscopy.

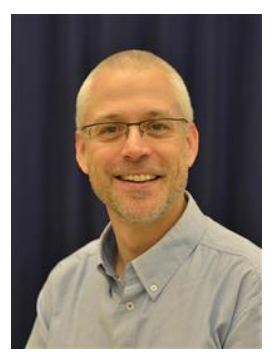

Peter E. Andersen received the MSc.E.E. and Ph.D. degrees from the Technical University of Denmark, Lyngby, Denmark in 1991 and 1994, respectively. $\mathrm{He}$ is currently a Senior Scientist and Research Professor at the Technical University of Denmark, where he leads the research within biomedical optics. He has more than 15 years of research experience with light sources for biomedical optics and optical coherence tomography systems and their application. He has coordinated several European research programs. He has authored or co-authored more than 125 scientific publications in the above-mentioned fields, respectively. He is the Deputy Editor of Optics Letters, editorial board member of the Journal of Biomedical Optics, and editorial board member of the Journal of Biophotonics, respectively. 